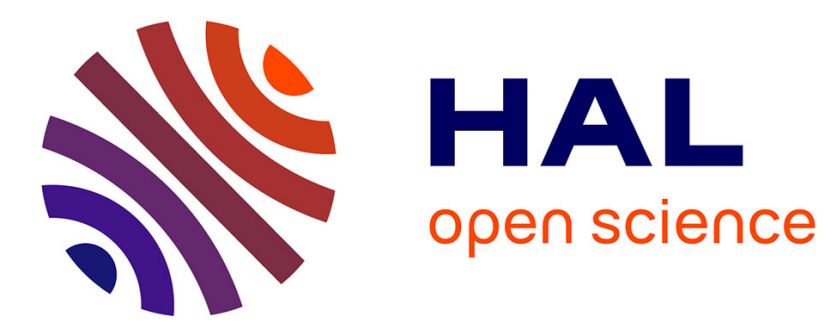

\title{
The influence of combustion SGS sub-models on the resolved flame propagation. Application to the LES of the Cambridge stratified flames.
}

\author{
Renaud Mercier, Thomas Schmitt, Denis Veynante, Benoit Fiorina
}

\section{To cite this version:}

Renaud Mercier, Thomas Schmitt, Denis Veynante, Benoit Fiorina. The influence of combustion SGS sub-models on the resolved flame propagation. Application to the LES of the Cambridge stratified flames.. Proceedings of the Combustion Institute, 2015, 35, pp.1259-1267. 10.1016/j.proci.2014.06.068 . hal-01219284

\section{HAL Id: hal-01219284 \\ https://hal.science/hal-01219284}

Submitted on 2 Mar 2020

HAL is a multi-disciplinary open access archive for the deposit and dissemination of scientific research documents, whether they are published or not. The documents may come from teaching and research institutions in France or abroad, or from public or private research centers.
L'archive ouverte pluridisciplinaire HAL, est destinée au dépôt et à la diffusion de documents scientifiques de niveau recherche, publiés ou non, émanant des établissements d'enseignement et de recherche français ou étrangers, des laboratoires publics ou privés. 


\title{
The influence of combustion SGS submodels on the resolved flame propagation. Application to the LES of the Cambridge stratified flames
}

\author{
R. Mercier*, T. Schmitt, D. Veynante, B. Fiorina \\ Ecole Centrale Paris, Grande Voie des Vignes, 92290 Châtenay-Malabry, France \\ CNRS, UPR 288, Laboratoire d'Energétique Moléculaire et Macroscopique, Combustion (EM2C), Grande Voie \\ des Vignes, 92290 Châtenay-Malabry, France
}

In Large Eddy Simulation (LES) of premixed and stratified combustion, the overall prediction of the flame consumption speed depends on various subgrid scale (SGS) submodels such as the flame wrinkling, the fuel stratification or heat losses. The objective of this study is to investigate the LES sensitivity to the submodeling strategies. Different heat losses and SGS flame wrinkling models are presented in the context of the Filtered TAbulated Chemistry for LES (F-TACLES) formulation. LES of the non-adiabatic nonswirling bluff-body stabilized Cambridge flames ( $\mathrm{SwB}$ burner) are presented. In this complex configuration, both flame brush and flow dynamics are influenced by flame consumption speed submodels. First, accounting for heat losses impacts the prediction of both velocity and temperature of the inner recirculation zone (IRZ). Second, model constants involved into SGS wrinkling submodels have a great impact on the mean flame brush position. The non-adiabatic formulation combined with a dynamic estimation of the SGS wrinkling model constant appears to be a very attractive approach and gives a very good prediction of both the mean flame location and the IRZ flow dynamics.

\section{Introduction}

Large Eddy Simulation of turbulent reactive flows is an attractive strategy to capture unsteady phenomena governing combustion dynamics and flame stabilization mechanisms. A first modeling approach is to describe the flame front as a propagating infinitely thin surface (G-equation formalism [1]). A second possibility consists in handling the flame thickness resolution using thickening $[2,3]$ or spatial filtering $[4,5]$ formalisms. These strategies differ in the chemical flame structure description, but all require the modeling of the subgrid scale turbulent flame consumption speed [6], which is strongly influenced by subgrid scale 
complex interactions between turbulence, chemistry, heat transfer and mixing. These phenomena are captured by dedicated submodels but their combined influences on the aerodynamic and flame brush predictions have not been investigated yet. The objective of this work is to assess the overall sensitivity of the reactive flow dynamics to the assumptions made to design these submodels.

Our study is conducted through several simulations of the non-swirling non-adiabatic stratified burner SwB [7], experimentally investigated at Cambridge University and at Sandia National Laboratories. Experimental campaigns reveal that the flame structure is influenced by fuel stratification, turbulence, heat losses and differential diffusion. The first part of this article describes the turbulent combustion model, named Filtered TAbulated Chemistry for LES (F-TACLES) $[5,8,9]$ and retained here for the numerical investigations. Submodels influencing the prediction of the subfilter flame consumption speed are discussed. The second part of the article presents a LES parametric study of the SwB configuration. The impact of heat losses on the flame stabilization process and temperature is discussed. Then, a sensitivity analysis is conducted to assess the influence of subgrid scale wrinkling modeling on the mean flame brush and flow dynamics predictions.

\section{Combustion model}

\subsection{Closure of the progress variable filtered equation}

In stratified combustion, the flame front is captured by the mixture fraction $z$ and the progress variable $Y_{c}$. Mixture fraction $z$, equal to 0 and 1 in oxidizer and fuel streams, respectively, is used to capture the fuel-air mixing. Progress variable $Y_{c}$, equal to 0 and $Y_{c}^{e q}(z)$ in fresh and burnt gases, respectively, tracks the flame front. The FTACLES formulation closes the filtered progress variable $\widetilde{Y}_{c}$ equation in flamelet regimes as $[5,8,9]$ :

$$
\begin{aligned}
\frac{\partial \bar{\rho} \widetilde{Y_{c}}}{\partial t}+\nabla \cdot\left(\bar{\rho} \widetilde{\mathbf{u}} \widetilde{Y_{c}}\right)= & \nabla \cdot\left(\Xi_{\Delta} \gamma \alpha_{Y_{c}}^{T a b} \rho_{0} D_{0} \nabla \widetilde{Y_{c}}\right) \\
& -\Xi_{\Delta} \gamma\left(\Omega_{Y_{c}}^{T a b}+\bar{\rho} \widetilde{\dot{\omega}}_{Y_{c}}^{T a b}\right)
\end{aligned}
$$

where $\rho$ and $\mathbf{u}$ are the flow density and velocity, respectively. Subscript 0 denotes reference quantities. By construction, this model propagates the resolved flame front at the subgrid scale turbulent flame speed $S_{T, \Delta}[8,9]$ :

$\rho_{0} S_{T, \Delta}=\Xi_{\Delta} \gamma \int_{0}^{1} \rho_{0} S_{l}^{a d}\left(z^{\prime}\right) P\left(z^{\prime}\right) d z^{\prime}$

where $S_{l}^{a d}(z)$ is the consumption speed [6] of a freely propagating adiabatic laminar premixed flame within fresh gases of mixture fraction $z, \Xi_{\Delta}$ the flame front wrinkling factor, measuring the subgrid scale flame surface and $\gamma$ a coefficient accounting for heat losses [9]. In the following, the mixture fraction Filtered Density Function (FDF) $P(z)$ is modeled by a $\beta$ function characterized by the filtered mixture fraction $\widetilde{z}$ and the subgrid scale mixture fraction variance $\widetilde{z^{\prime \prime 2}}$.

The functions $\alpha_{Y_{c}}^{T a b}, \Omega_{Y_{c}}^{T a b}$ and $\widetilde{\dot{\omega}}_{Y_{c}}^{T a b}$ in Eq. (1) are designed to model the unresolved contributions to molecular diffusion, convection and chemical reaction, respectively. They are tabulated by filtering 1-D adiabatic premixed flame elements computed including detailed chemistry and complex transport and stored as a function of four parameters: $\widetilde{Y}_{c}, \widetilde{z}, \widetilde{z^{\prime \prime 2}}$ and the filter scale $\Delta[8,9] . \Delta$ is chosen so that the reaction layer thickness (based on $\widetilde{\dot{\omega}}_{Y_{c}}^{T a b}$ ) is resolved on a minimum of 4 nodes [10] leading to $\Delta \approx 5 \Delta_{x}$ where $\Delta_{x}$ denotes the cell size. Balance equations for the filtered mixture fraction $\widetilde{z}$ and the mixture fraction variance $\widetilde{z^{\prime \prime 2}}[8]$ are solved in addition to the $\widetilde{Y}_{c}$ balance equation.

\subsection{Modeling dependencies of $S_{T, \Delta}$}

Equation (2) evidences that modeling choices for $\Xi_{\Delta}, \gamma$ and $P\left(z^{\prime}\right)$ directly influence the flame consumption speed. The role of $P\left(z^{\prime}\right)$ was discussed previously [8] and the present work focusses on the modeling of the subgrid scale flame wrinkling $\Xi_{\Delta}$ and the heat loss correction $\gamma$ factors.

\subsubsection{Modeling the influence of heat losses}

The impact of heat losses is accounted for through the correction factor $\gamma$ modeled as [9]:

$\gamma=\frac{\int_{0}^{1} \rho_{0} S_{l}\left(z^{\prime}, \Delta \widetilde{h}\right) P\left(z^{\prime}\right) d z^{\prime}}{\int_{0}^{1} \rho_{0} S_{l}^{a d}\left(z^{\prime}\right) P\left(z^{\prime}\right) d z^{\prime}}$

where $\Delta \widetilde{h}$, the enthalpy defect relative to the fresh gases, is defined as $\Delta \widetilde{h}=h^{a d}(\widetilde{z})-\widetilde{h} \cdot h^{a d}$ denotes the adiabatic (sensible plus chemical) enthalpy and only depends on mixture fraction and fresh gas temperature while $\widetilde{h}$ is the resolved enthalpy accounting for heat loss. $S_{l}\left(z^{\prime}, \Delta \widetilde{h}\right)$, the laminar consumption speed at a given $\Delta h$, is estimated from burner-stabilized 1-D flames [11]. The heat loss correction factor $\gamma$ is computed from both the 1-D non-adiabatic flame thermochemical structure and the mixture fraction FDF and does not necessitate any ad hoc parametrization. $\gamma=1$ in adiabatic flows and decreases when heat losses slow down the combustion chemistry. The limit $\gamma=0$ corresponds to flame quenching.

\subsubsection{Modeling the subgrid scale flame wrinkling}

The subgrid scale flame wrinkling model is based on the expression initially proposed by Charlette et al. [12] and later improved [13]: 
$\Xi_{\Delta}=\left(1+\min \left[\frac{\Delta}{\delta_{l}^{0}}-1, \Gamma_{\Delta}\left(\frac{\Delta}{\delta_{l}^{0}}, \frac{u_{\Delta}^{\prime}}{S_{l}^{0}}, R e_{\Delta}\right) \frac{u_{\Delta}^{\prime}}{S_{l}^{0}}\right]\right)^{\beta}$

where $\operatorname{Re}_{\Delta}=\left(u_{\Delta}^{\prime} \Delta\right) / v$ and $u_{\Delta}^{\prime}$ are the subgrid scale Reynolds number and turbulence intensity, respectively, while $\delta_{l}^{0}$ is the laminar flame thickness. $\beta$ is a model parameter. The efficiency function $\Gamma_{\Delta}$, which estimates the net straining effect of all turbulent scales smaller than $\Delta$, is modeled as in [12]. Note that other modeling strategies are possible as discussed in $[14,15]$. Without SGS turbulence, the flame wrinkling is fully resolved $\left(\Xi_{\Delta} \rightarrow 1\right.$ when $u_{\Delta}^{\prime} \rightarrow 0$ ). On the other hand, for large turbulence intensities, the sub-grid scale wrinkling factor $\Xi_{\Delta}$ reduces to the fractal-like power-law:

$\Xi_{\Delta}=\left(\Delta / \delta_{l}^{0}\right)^{\beta}$

Both the estimations of the parameter $\beta$ (Eqs. (4) and (5)) and the subgrid scale velocity fluctuations $u_{\Delta}^{\prime}$ (Eq. (4)) require additional submodels discussed bellow

\section{Estimation of $\beta$.}

Various values of $\beta$ have been reported in the literature depending on the flame and flow topology as well as the turbulence intensity [16]. For instance, $\beta=0.5$ produces reasonable behavior of SGS wrinkling model over a significant range of flame and turbulence parameters [12,16], but $\beta=0.3$ is also often encountered in the literature $[13,17-20]$.

An alternative is to automatically adjust $\beta$ from the known resolved scales [13,16,20-22]. Following the original idea of the Germano et al. dynamic model [23] for unresolved momentum transport, the $\beta$ parameter is expressed by equating the flame surface averaged over a given volume, referred as $\langle\cdot\rangle$ when estimated following two routes: $(i)$ filtering the LES total flame surface density (i.e. flame surface per unit volume) at the testfilter scale $\widehat{\Delta}$; (ii) filtering the resolved progress variable at the scale $\widehat{\Delta}$ to estimate the total flame surface density at the effective filter scale $\widehat{\bar{\Delta}}=\sqrt{(\Delta)^{2}+(\widehat{\Delta})^{2}}$ obtained when combining LES and test Gaussian filters (see [20] for details):

$$
\left(\frac{\Delta}{\delta_{l}^{0}}\right)^{\beta}\langle|\widehat{\nabla \widetilde{c}}|\rangle=\left(\frac{\widehat{\bar{\Delta}}}{\delta_{l}^{0}}\right)^{\beta}\langle|\nabla \widehat{\widetilde{c}}|\rangle
$$

where $\widehat{Q}$ denotes test-filtering of the quantity $Q$, $\widehat{\widetilde{Q}}$ denotes test-filtering of the resolved quantity $\widetilde{Q}$ and $\langle\cdot\rangle$ averages over a volume, here transversal slices of thickness $4 \Delta$. The normalized progress variable $\widetilde{c}$ is defined from the resolved progress variable $\widetilde{Y_{c}}$ as $\widetilde{c}=\widetilde{Y_{c}} / Y_{c}^{\mathrm{eq}}(\widetilde{z})$. The wrinkling factor is modeled following the strategy prescribed in [22] where the flame wrinkling cutoff scale $\delta_{c}$ is set to two times the thermal flame thickness $\delta_{l}^{0}$ and the $\beta$ parameter is given by Eq. (6):

$$
\beta=\frac{\ln (\langle|\widehat{\nabla \widetilde{c}}|\rangle /\langle|\nabla \widehat{\widetilde{c}}|\rangle)}{\ln (\widehat{\bar{\Delta}} / \Delta)}
$$

Estimation of $u_{\Delta}^{\prime}$. The Prandtl-Kolmogorov approach [24,25], consists in estimating $u_{\Delta}^{\prime}$ from the turbulent viscosity $v_{t}$ closing subgrid scale turbulent transport:

$u_{\Delta}^{\prime}=\sqrt{2 / 3}\left(v_{t} / C_{k} \Delta_{x}\right)\left(\Delta / \Delta_{x}\right)^{1 / 3}$

The value of the model constant $C_{k}$ is estimated in ideal cases as homogeneous and isotropic turbulence or highly sheared mixing layers [26]. Two typical values $\left(C_{k}=0.05\right.$ and $\left.C_{k}=0.1\right)$ are considered here. Unfortunately, this expression does not guaranty $u_{\Delta}^{\prime}=0$ when the flame is not wrinkled by turbulence.

An alternative is to define an operator which subtracts the dilatational part of the resolved velocity field [2]:

$u_{\Delta}^{\prime}=c_{2} \Delta_{x}^{3}\left|\nabla^{2}(\nabla \times \widetilde{\mathbf{u}})\right|\left(\Delta /\left(n_{x} \Delta_{x}\right)\right)^{1 / 3}$

where $n_{x}=10$ and $c_{2}=2$ are model constants. This formulation degenerates towards laminar flame regimes.

The influence of the three submodels for $\gamma, \beta$ and $u_{\Delta}^{\prime}$ on the simulation of a non-adiabatic turbulent stratified flame are identified in the following sections.

\section{Experimental and numerical set-up of the Cam- bridge stratified burner}

The Cambridge stratified swirl burner (SwB) $[7,27]$ is composed of two concentric tubes surrounding a central bluff-body. The burner dimensions and the different injected streams are illustrated in Fig. 1. The inner (i) and outer (o) methane-air streams inlets are controlled independently in terms of bulk velocity $\bar{U}$ and equivalence ratio $\phi$. A surrounding air coflow of velocity $\overline{U_{c o}}$ isolates the flame from ambient perturbations. The injected streams temperature is $T_{0}=298.0$

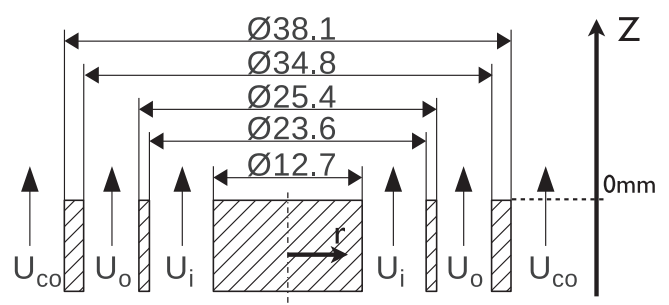

Fig. 1. Cross section of the axisymmetric SwB burner. Diameters are given in $\mathrm{mm}$. 
Table 1

Operating conditions.

\begin{tabular}{llllll}
\hline Case & $\phi_{i}[-]$ & $\phi_{o}[-]$ & $\overline{U_{i}}\left[\frac{m}{s}\right]$ & $\overline{U_{o}}\left[\frac{m}{s}\right]$ & $\overline{U_{c o}}\left[\frac{m}{s}\right]$ \\
\hline SwB1 & 0.75 & 0.75 & 8.31 & 18.7 & 0.4 \\
SwB5 & 1.0 & 0.5 & 8.31 & 18.7 & 0.4 \\
\hline
\end{tabular}

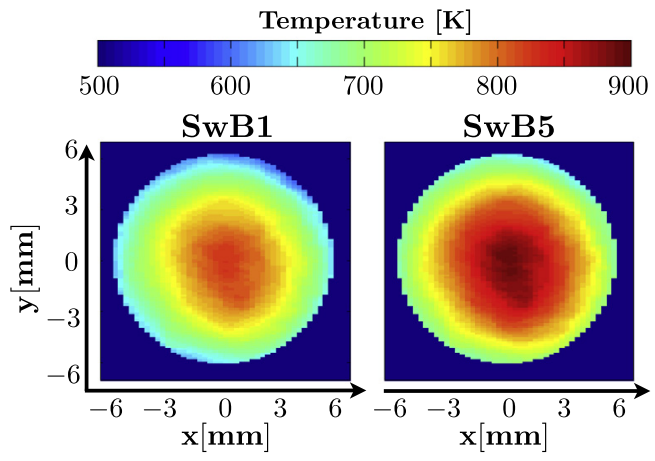

Fig. 2. Measured bluff-body surface temperatures for SwB1 and SwB5 cases [28].

K. A wide range of operating conditions have been experimentally investigated by varying the degree of stratification and swirl. The numerical work focuses here on two non-swirling cases for which operating conditions are indicated in Table 1: the premixed reactive configuration SwB1 and the stratified reactive configuration SwB5. Experimental data available include velocity, temperature and local equivalence ratio [7,27]. Recent measurements of the bluff-body wall temperature using phosphor thermometry [28], shown in Fig. 2, evidences the importance of heat transfer from burnt gases to the wall.

LES are performed using the YALES2 lowMach number, unstructured finite volume flow solver [29]. Fourth-order schemes are used for both spatial discretization and time integration. The SGS turbulence is described by the $\sigma$-model [30]. Chemistry is tabulated from a collection of 1-D laminar premixed flames computed with the

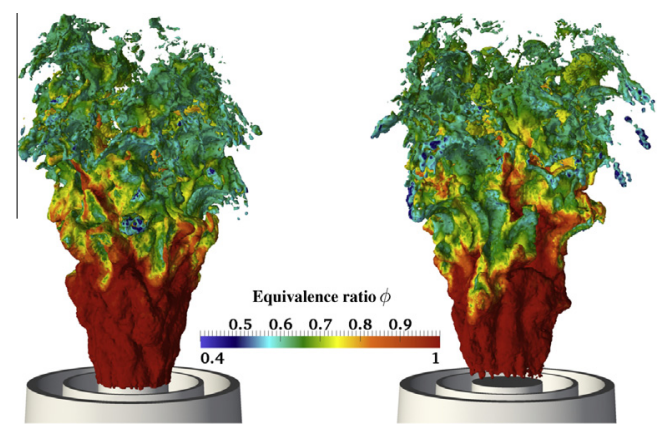

Fig. 3. Isosurface of filtered progress variable reaction rate $\bar{\rho} \widetilde{\tilde{\omega}}_{Y_{c}}$ colored by fresh gas equivalence ratio $\phi$ for the stratified (SwB5) case. Left: Adiabatic computation (5-AD-CH1). Right: non-adiabatic computation (5NAD-CH1).

detailed chemistry scheme proposed by Lindstedt [31], involving 29 species and 141 reactions. The tabulated chemistry is coupled to the LES balance equations with the non-adiabatic stratified FTACLES model introduced in Section 2.1 and detailed in [9]. Homogeneous and isotropic turbulence [32] is injected into the tubes at $\mathrm{Z}=$ $-120 \mathrm{~mm}$ and is adjusted to match velocity fluctuations profiles measured at $Z=2 \mathrm{~mm}(Z=0 \mathrm{~mm}$ being the plane located at the burner exit). The turbulent intensity is set to $10 \%$ while the integral length scale is $L_{e} \approx D_{h} / 4 \approx 3.0 \mathrm{~mm}$ where $D_{h}$ denotes the hydraulic diameter. Preliminary simulations of the non-reacting case (not show here due to space limitation), performed on two meshes (6.2 and 17.6 million nodes), have shown that the coarser grid was sufficient to capture $\mathrm{SwB}$ flow dynamics. The coarser grid, used for this study, has been designed to be representative of industrial applications. The characteristic mesh size in the flame region is $\Delta_{x} \approx \delta_{l}^{0}$ ensuring a major contribution of the combustion model in the LES. A parametric study has then been performed on reacting cases through 9 numerical simulations whose characteristics are summarized in Table 2 and are analyzed below.

Table 2

Simulated cases. The flow-through-time (FTT) is estimated as FTT $=\mathrm{L} / \overline{U_{i}}=10 \mathrm{~ms}$ with $\mathrm{L}=0.1 \mathrm{~m}$ the height of the reaction zone and $\overline{U_{i}}=10 \mathrm{~m} \cdot \mathrm{s}^{-1}$ the bulk velocity of inner tube. The F-TACLES filtered look-up tables are generated with a flame filter size $\Delta=2.5 \mathrm{~mm}$. These computations are conducted on an IBM Blue Gene/Q machine on 1024 cores.

\begin{tabular}{llllllll}
\hline Simu. \# & $\begin{array}{l}\text { Exp. } \\
\text { config }\end{array}$ & $\mathrm{N}_{\text {nodes }}$ & $\mathrm{T}_{\text {bluff-body }}$ & $\Xi_{\Delta}$ Model & $u_{\Delta}^{\prime}$ Model & $\beta$ Model & $\begin{array}{l}\text { 1FTT cost } \\
\text { [hCPU] }\end{array}$ \\
\hline 1-AD-CH1 & SwB1 & $6.2 \cdot 10^{6}$ & Adiabatic & Eq. (4) & Eq. $(8)\left(C_{k}=0.1\right)$ & 0.5 & $16800 \mathrm{hCPU}$ \\
1-NAD-CH1 & SwB1 & $6.2 \cdot 10^{6}$ & Expe. [28] & Eq. (4) & Eq. (8) $\left(C_{k}=0.1\right)$ & 0.5 & $16900 \mathrm{hCPU}$ \\
5-AD-CH1 & SwB5 & $6.2 \cdot 10^{6}$ & Adiabatic & Eq. (4) & Eq. $(8)\left(C_{k}=0.1\right)$ & 0.5 & $18500 \mathrm{hCPU}$ \\
5-NAD-CH1 & SwB5 & $6.2 \cdot 10^{6}$ & Expe. [28] & Eq. (4) & Eq. (8) $\left(C_{k}=0.1\right)$ & 0.5 & $18800 \mathrm{hCPU}$ \\
5-NAD-CH2 & SwB5 & $6.2 \cdot 10^{6}$ & Expe. [28] & Eq. (4) & Eq. (8) $\left(C_{k}=0.05\right)$ & 0.5 & $18800 \mathrm{hCPU}$ \\
5-NAD-CO & SwB5 & $6.2 \cdot 10^{6}$ & Expe. [28] & Eq. (4) & Eq. (9) & 0.5 & $19000 \mathrm{hCPU}$ \\
5-NAD-B5 & SwB5 & $6.2 \cdot 10^{6}$ & Expe. [28] & $\Xi_{\Delta}=\left(\Delta / \delta_{l}\right)^{\beta}$ & - & 0.5 & $18400 \mathrm{hCPU}$ \\
5-NAD-B3 & SwB5 & $6.2 \cdot 10^{6}$ & Expe. [28] & $\Xi_{\Delta}=\left(\Delta / \delta_{l}\right)^{\beta}$ & - & 0.3 & $18400 \mathrm{hCPU}$ \\
5-NAD-BD & SwB5 & $6.2 \cdot 10^{6}$ & Expe. [28] & $\Xi_{\Delta}=\left(\Delta / \delta_{c}\right)^{\beta}$ & - & Dynamic $[20,22]$ & $22300 \mathrm{hCPU}$ \\
\hline
\end{tabular}




\section{Result analysis}

\subsection{Impact of heat losses on flame stabilization and temperature field}

For both SwB1 and SwB5 cases, two simulations are conducted either by assuming burner adiabaticity or by imposing measured bluff-body temperature shown in Fig. 2 as thermal boundary conditions (Table 2). These four computations use the same subgrid scale flame wrinkling closure. Figure 3 compares two instantaneous views of the filtered progress variable reaction rate iso-surface extracted from adiabatic and non-adiabatic simulations of the SwB5 case. The flame is anchored on the burner bluff-body for adiabatic conditions while the non-adiabatic simulation predicts a lifted flame. Indeed, heat losses affect the flow enthalpy and influence the turbulent filtered flame propagation speed $[33,34]$ through the factor $\gamma$ (Eq. (2)). Figure 4 compares mean temperature radial profiles against experimental data for both SwB1 and SwB5 cases. Two axial distances from the burner exit, corresponding to the bluff-body inner recirculation zone (IRZ), are selected. As expected, the adiabatic simulation of SwB5 flame overestimates the burnt gases temperature for $r<6 \mathrm{~mm}$ by about $150 \mathrm{~K}$ while the non-adiabatic simulation, accounting for the IRZ cooling at the bluff-body wall surface, correctly captures it. However, this tendency is not verified in the SwB1 case, where the adiabatic LES predicts surprisingly well the IRZ temperature which is now underestimated by the non-adiabatic LES. This phenomenon is explained by comparing computed and measured equivalence ratio in Fig. 5. As discussed in [35], experiments revealed that atom balances (atomic mass

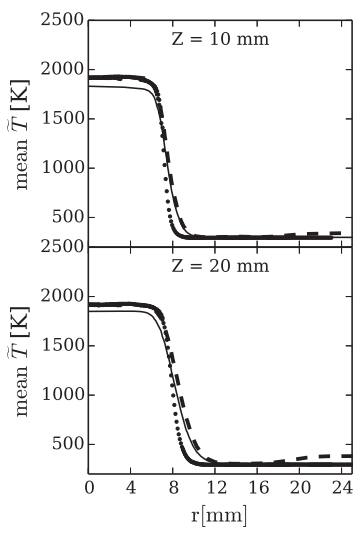

(a) Premixed/SwB1 case

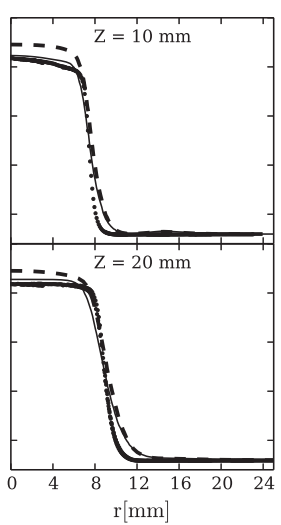

(b) Stratified/SwB5 case
Fig. 4. Mean temperature $\widetilde{T}$ radial profiles. Legend: - - adiabatic computations (1-AD-CH1 and 5-AD-CH1); - non-adiabatic computations (1-NAD-CH1 and 5NAD-CH1); •• experiments.

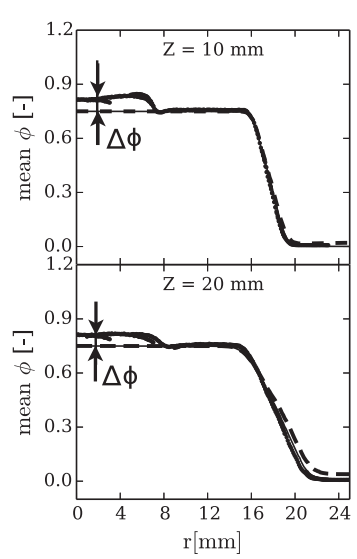

(a) Premixed/SwB1 case

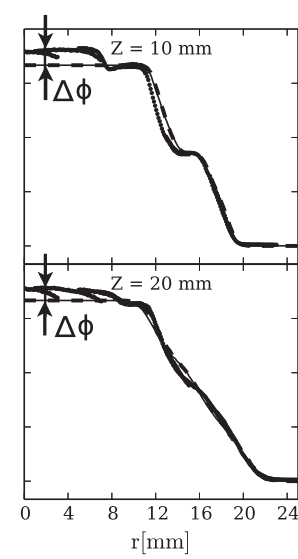

(b) Stratified/SwB5 case
Fig. 5. Mean fresh gas equivalence ratio $\phi$ radial profiles. Legend: - - - adiabatic computations (1-AD$\mathrm{CH} 1$ and 5-AD-CH1); - non-adiabatic computations (1-NAD-CH1 and 5-NAD-CH1); •• experiments.

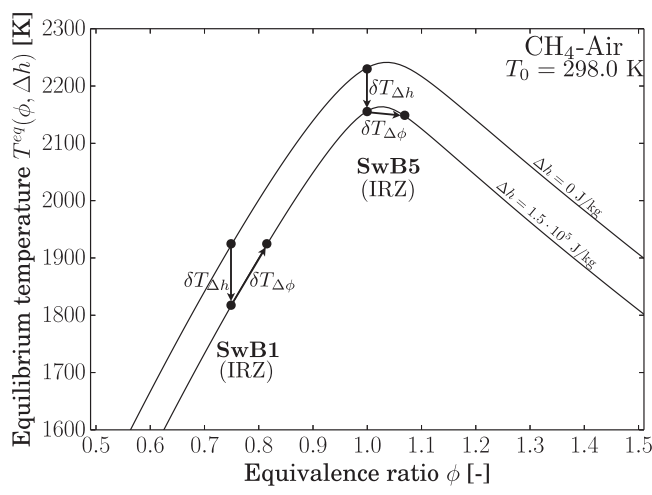

Fig. 6. Equilibrium temperature function of fresh gas equivalence ratio $\phi$ for adiabatic and non-adiabatic situations. $\mathrm{CH}_{4}$-Air fresh gas temperature is taken equal to $T_{0}=298.0 \mathrm{~K}$. Legend: upper curve: adiabatic $(\Delta h=0)$; lower curve: non-adiabatic $\left(\Delta h=1.5 \cdot 10^{5}\right.$ $\mathrm{J} / \mathrm{kg})$.

fractions) were not conserved across the flame brush going from reactants to products. Equilibrium temperature is consequently affected by this elemental composition variation. This complex phenomenon is not captured by the F-TACLES model where chemistry is tabulated from 1-D premixed flamelets, neglecting therefore complex transport across iso-equivalence ratio surfaces [36]. This effect could be handled by adding a coordinate to the chemical database [37,38], a task out of the scope of the present study. An a priori estimation of the error induced by such assumption in both SwB1 and SwB5 is nevertheless proposed. Figure 6, plots the equilibrium temperature as a function of the equivalence ratio, for 
two different enthalpy defects $\Delta h . \Delta h=0$ corresponds to the adiabatic equilibrium temperature while $\Delta h=1.5 \cdot 10^{5} \mathrm{~J} / \mathrm{kg}$ is the mean enthalpy defect extracted from the centerline of the IRZ in non-adiabatic computations. The variation of equivalence ratio $\Delta \phi$ due to differential diffusion is estimated from experimental data. $\Delta \phi$ is shown in Fig. 5 for both cases SwB1 and SwB5. The temperature variations $\delta T_{\Delta h}$ and $\delta T_{\Delta \phi}$ due to heat losses and differential diffusion, respectively, are identified for both SwB1 and SwB5. As the IRZ mixture of SwB5 is almost stoichiometric, it is observed in Fig. 6 that $\delta T_{\Delta h}>\delta T_{\Delta \phi}$, explaining why neglecting differential diffusion across isoequivalence ratio will not lead to significant departure between measured and predicted temperature (Fig. 4 right). At the opposite, $\delta T_{\Delta h} \approx \delta T_{\Delta \phi}$ for the lean fully premixed SwB1 case. Then, the non-adiabatic simulation without accounting for complex transport effect cannot capture accurately the IRZ temperature. The SwB1 adiabatic simulation actually predicts fairly well the burnt gases temperature (Fig. 4 left) because of two compensating errors.

\subsection{Impact of the subgrid scale wrinkling model on the flame brush position}

The wrinkling of resolved flame surface shown in Fig. 3 evolves along the centerline: the flame front is weakly affected by turbulent motions near the burner exit whereas the reactive layer is strongly wrinkled by the turbulent shear layer further downstream. Assuming similarity between resolved and SGS wrinkling scales, the same complex behavior is expected for $\Xi_{\Delta}$. Different modeling strategies for $\Xi_{\Delta}$ are now challenged on the stratified SwB5 case, for which the IRZ thermochemical properties are well predicted. Heat transfer to the wall are systematically included in the following simulations.

The flame wrinkling model given by Eq. (4) is used in simulations 5-NAD-CH1, 5-NAD-CH2 and 5-NAD-CO (Table 2). These computations only differ by the SGS velocity fluctuation model. 5-NAD-CH1 and 5-NAD-CH2 estimate $u_{\Delta}^{\prime}$ from the Prandtl-Kolmogorov approach (Eq. (8)) with $C_{k}=0.1$ and $C_{k}=0.05$, respectively. 5-NAD-CO retains the Colin model (Eq. (9)). Mean temperature profiles are compared to experimental data in Fig. 7. 5-NAD-CH2 mispredicts the mean flame brush position for axial distances $Z \geqslant 20 \mathrm{~mm}$. However, 5-NAD-CH1 predicts a flame brush closer to experimental measurements showing that the results are very sensitive to the PrandtlKolmogorov model constant $C_{k}$ in Eq. (8). The 5-NAD-CO simulation also gives a good prediction of the mean flame brush but the same sensitivity to the $u_{\Delta}^{\prime}$ model constant is expected. Prediction of the turbulent flame position is then strongly correlated to the $u_{\Delta}^{\prime}$ submodel uncertainties.

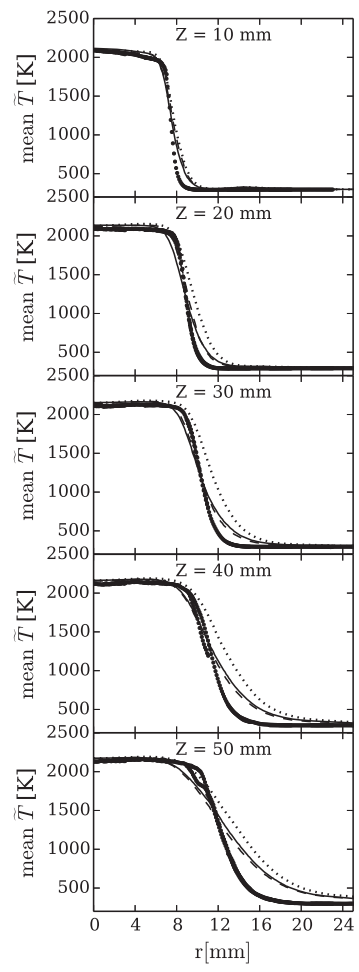

(a) Impact of $u_{\Delta}^{\prime}$

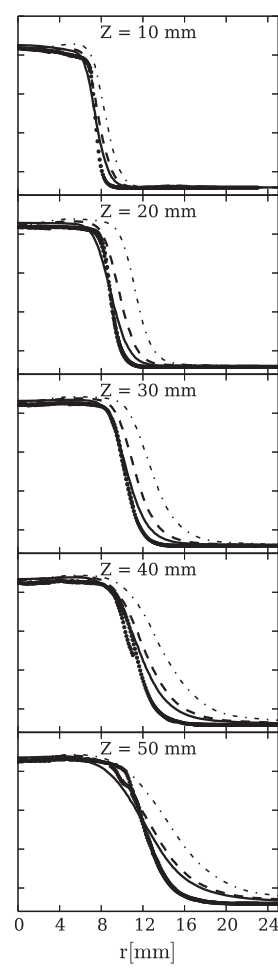

(b) Impact of $\beta$
Fig. 7. Mean temperature $\widetilde{T}$ radial profiles for the stratified case (SwB5) case. Left plot: - 5-NAD-CH1; 5-NAD-CH2; - - 5-NAD-CO; • • Experiments. Right plot: - . - 5-NAD-B5; - - - 5-NAD-B3; - - 5-NAD-BD; • • experiments.

The influence of the $\beta$ parameter is investigated on the saturated $\Xi_{\Delta}$ formulation (Eq. (5)). Two simulations, namely 5-NAD-B5 and 5-NAD-B3, have been performed with $\beta=0.5$ and $\beta=0.3$, respectively. The saturated formulation of $\Xi_{\Delta}$ is not relevant to predict unity SGS flame wrinkling in laminar regimes. This is why neither 5-NADB5 or 5-NAD-B3 solution capture the flame brush position at first axial locations. However, the 5NAD-B3 leads to fair agreement with experimental data further downstream whereas 5-NAD-B5 completely mispredict the mean flame position. The constant model parameter $\beta$ can then be adjusted to fit either upstream or downstream flame front wrinkling. As shown by plots of 5-NAD-BD simulation, dynamic estimation of $\beta$ brings then enough flexibility to Eq. (5) for capturing both quasi-laminar and turbulent flame regimes. It provides a very good prediction of the mean properties of the flame both at the quasi-laminar flame basis ${ }^{1}(\beta=0.2$ at

\footnotetext{
${ }^{1}$ The dynamic estimation of the $\beta$ parameter goes to zero in laminar cases or when the wrinkling is fully resolved as shown in [20].
} 


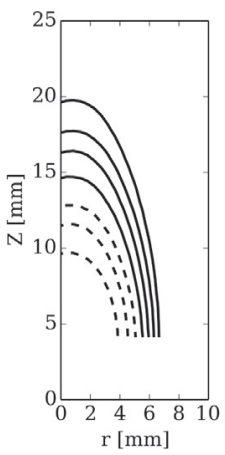

(a) Experiments (2-D PIV)

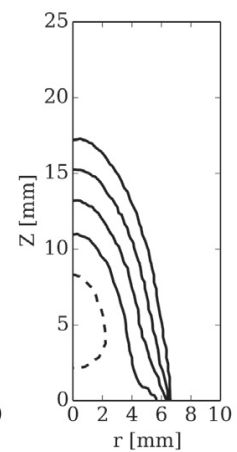

(b) 5-AD- $\mathrm{CH} 1$

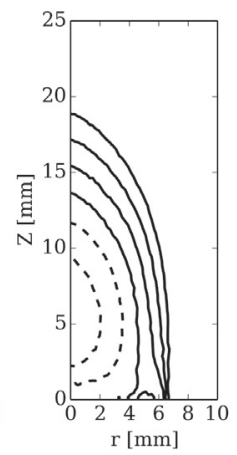

(c) 5-NAD-CH1

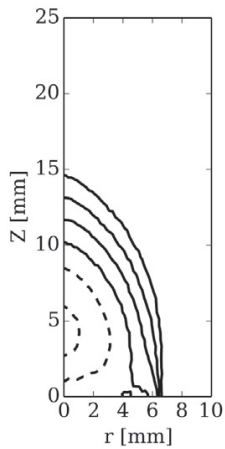

(d) 5-NAD-CH2

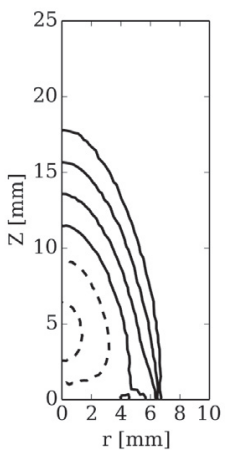

(e) 5-NAD-CO

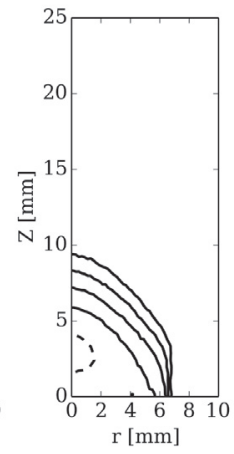

(f) 5-NAD-B5

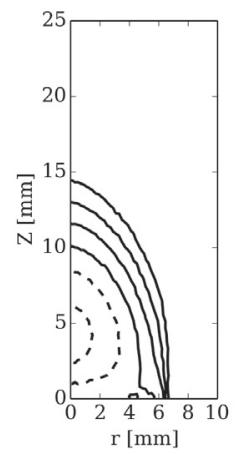

(g) 5-NAD-B3

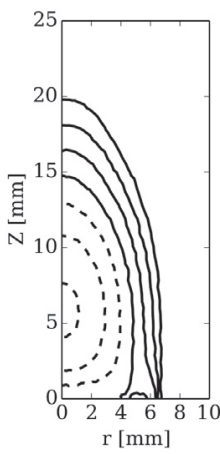

(h) 5-NAD-BD

Fig. 8. Cross section in the medium plane of the iso-surfaces of mean axial velocity $\widetilde{U_{z}}$ for the stratified case (SwB5). For each figure, isolines values are, from right to left, $\widetilde{U_{z}}=\{3 ; 2 ; 1 ; 0 ;-1 ;-2 ;-3\} \mathrm{m} . \mathrm{s}^{-1}$. Dashed lines: $\widetilde{U_{z}}<0 ;$ solid lines: $\widetilde{U}_{z} \geqslant 0$.

$\mathrm{Z}=10 \mathrm{~mm})$ and further downstream where the flame is fully turbulent $(\beta=0.8$ at $Z=30 \mathrm{~mm}$ ). The dynamic estimation of the $\beta$ parameter only induces a $20 \%$ increase in computational cost. All radial profiles of mean temperature exhibit a broader flame brush than the measured one far from the burner exit. This result may be due to the azimuthal averaging operation as discussed in.

\subsection{Influence of turbulent combustion submodels on the flow dynamics}

Figure 8 compares medium cross section of mean axial velocity iso-surfaces at the IRZ location for the SwB5 case. First, both the position and the dynamics of the IRZ differ between 5-AD-CH1 and 5-NAD-CH1 as thermal expansion is reduced near the burner wall when heat losses are considered. The non-adiabatic simulation 5-NAD-CH1 predicts a faster rotation of the recirculation zone evidenced by a larger absolute value of the negative axial velocity. Second, non-adiabatic simulations evidence a direct correlation between the height of the IRZ (Fig. 8) and the mean flame brush position near the burner exit (Fig. 7): the highest opening angles of the mean flame brush correspond to the smallest recirculation zones. Same conclusions are made comparing axial and radial velocity profiles to experimental data at different distances from burner exit (not shown here due to space limitation). Therefore, subgrid scale wrinkling models also impact the IRZ dynamics. In particular, it is worth noting that 5-NAD-BD gives a good prediction of both the mean flame brush position and the IRZ aerodynamic.

\section{Conclusion}

A sensitivity study has been conducted on the non-swirling SwB flames. Two modeling issues have been considered. First, heat losses are found to decrease the temperature of burnt gas in the IRZ improving the comparison with experiments. It also drastically decreases the flame consumption speed at the flame basis which consequently influences the flow through thermal expansion. The $\Xi_{\Delta}$ models sensitivity analysis shows that the saturated formulation (Eq. (5)) with a static estimation of the $\beta$ parameter is too rough to capture different flame wrinkling regimes. The 
Charlette et al. [12] model (Eq. (4)) is theoretically attractive as designed to degenerate correctly towards laminar flame regime but is very sensitive to model parameters such as the SGS velocity fluctuations $u_{\Delta}^{\prime}$. Finally, the dynamic formulation is sufficiently flexible to capture the flame brush position over the whole computational domain. Similar conclusions may be achieved with any model based on flame surface considerations and also depending on sub-models for the flame consumption speed.

\section{Acknowledgments}

The authors thank Prof. Simone Hochgreb for providing experimental data. Vincent Moureau and Ghislain Lartigue from the CORIA lab, and the SUCCESS scientific group are acknowledged for providing the YALES2 code. This work was supported by the ANR-10-EESI-05 Grant of the French Ministry of Research and was performed using HPC resources from GENCI-IDRIS (Grant 2013-x20132b0164).

\section{References}

[1] H. Pitsch, Combust. Flame 143 (4) (2005) 587-598.

[2] O. Colin, F. Ducros, D. Veynante, T. Poinsot, Phys. Fluids 12 (7) (2000) 1843-1863.

[3] F. Proch, A. M. Kempf, Combust. Flame, 2014, in press, http://dx.doi.org/10.1016/j.combustflame. 2014.04.010.

[4] C. Duwig, Combust. Theory Model. 13 (2) (2009) 251-268.

[5] B. Fiorina, R. Vicquelin, P. Auzillon, N. Darabiha, O. Gicquel, D. Veynante, Combust. Flame 157 (3) (2010) 465-475. http://dx.doi.org/10.1016/ j.combustflame.2009.09.015.

[6] T. Poinsot, D. Veynante, Theoretical and Numerical Combustion, third ed., 2012.

[7] M.S. Sweeney, S. Hochgreb, M.J. Dunn, R.S. Barlow, Proc. Combust. Inst. 33 (1) (2011) 14191427. http://dx.doi.org/10.1016/j.proci.2010.05.069.

[8] P. Auzillon, O. Gicquel, N. Darabiha, D. Veynante, B. Fiorina, Combust. Flame 159 (8) (2012) 2704 2717. http://dx.doi.org/10.1016/j.combustflame. 2012.03.006.

[9] R. Mercier, P. Auzillon, V. Moureau, N. Darabiha, O. Gicquel, D. Veynante, B. Fiorina, Flow Turbul. Combust. in press. http://dx.doi.org/10.1007/ s10494-014-9550-8.

[10] P. Auzillon, B. Fiorina, R. Vicquelin, N. Darabiha, O. Gicquel, D. Veynante, Proc. Combust. Inst. 33 (1) (2011) 1331-1338. http://dx.doi.org/10.1016/ j.proci.2010.05.045.

[11] B. Fiorina, R. Baron, O. Gicquel, D. Thévenin, S. Carpentier, N. Darabiha, Combust. Theory Model. 7 (3) (2003) 449-470. http://dx.doi.org/10.1088/ $1364-7830 / 7 / 3 / 301$.

[12] F. Charlette, C. Meneveau, D. Veynante, Combust. Flame 131 (1/2) (2002) 159-180. http://dx.doi.org/ 10.1016/S0010-2180(02)00401-7.
[13] G. Wang, M. Boileau, D. Veynante, Combust. Flame 158 (11) (2011) 2199-2213. http:// dx.doi.org/10.1016/j.combustflame.2011.04.008.

[14] A. De, S. Acharya, Combust. Sci. Technol. 181 (10) (2009) 1231-1272. http://dx.doi.org/10.1080/ 00102200903076266.

[15] E.R. Hawkes, O. Chatakonda, H. Kolla, A.R. Kerstein, J.H. Chen, Combust. Flame 159 (8) (2012) 2690-2703. http://dx.doi.org/10.1016/j. combustflame.2011.11.020.

[16] F. Charlette, C. Meneveau, D. Veynante, Combust. Flame 131 (1/2) (2002) 181-197. http://dx.doi.org/ 10.1016/S0010-2180(02)00401-7.

[17] F. Gouldin, Combust. Flame 68 (3) (1987) 249-266, http://dx.doi.org/10.1016/0010-2180(87)90003-4.

[18] A. Kerstein, Combust. Sci. Technol. 60 (4-6) (1988) 441-445. http://dx.doi.org/10.1080/ 00102208808923997.

[19] T. Schmitt, A. Sadiki, B. Fiorina, D. Veynante, Proc. Combust. Inst. 34 (1) (2013) 1261-1268. http://dx.doi.org/10.1016/j.proci.2012.06.150.

[20] D. Veynante, T. Schmitt, M. Boileau, V. Moureau, in: Proceedings of the Summer Program 2012, Center For Turbulence Research, Stanford University, 2013.

[21] G. Wang, M. Boileau, D. Veynante, K. Truffin, Combust. Flame 159 (8) (2012) 2742-2754. http:// dx.doi.org/10.1016/j.combustflame.2012.02.018.

[22] T. Schmitt, M. Boileau, D. Veynante, V. Moureau, in: Eighth International Symposium On Turbulence and Shear Flow Phenomena, 2013.

[23] M. Germano, U. Piomelli, P. Moin, W.H. Cabot, Phys. Fluids 3 (7) (1991) 1760-1765. http:// dx.doi.org/10.1063/1.857955.

[24] L. Prandtl, Akad. Wiss. Göttingen, Math. Phys. (619).

[25] A.N. Kolmogorov, lzv. Akad. Nauk, USSR, Seria fizicheska VI (1-2) (1942) 56-58.

[26] W. Rodi, J. Fluids Eng. 97 (3) (1975) 386-389. http://dx.doi.org/10.1115/1.3447325.

[27] M.S. Sweeney, S. Hochgreb, R.S. Barlow, Combust. Flame 158 (5) (2011) 935-948. http://dx.doi.org/ 10.1016/j.combustflame.2011.02.007.

[28] M. Euler, R. Zhou, S. Hochgreb, A. Dreizler, in: Proceedings of the European Combustion Meeting, 2013.

[29] V. Moureau, P. Domingo, L. Vervisch, Comptes Rendus Mécanique 339 (2-3) (2011) 141-148. http:// dx.doi.org/10.1016/j.crme.2010.12.001.

[30] F. Nicoud, H.B. Toda, O. Cabrit, S. Bose, J. Lee, Phys. Fluids 23 (8) (2011) 085106. http://dx.doi.org/ $10.1063 / 1.3623274$.

[31] P. Lindstedt, Tech. Rep. TR-96 009, Brite Euram Program Project BE 951523 (1997).

[32] T. Passot, A. Pouquet, J. Fluid Mech. 181 (1987) 441466. http://dx.doi.org/10.1017/S0022112087002167.

[33] A. Ketelheun, G. Kuenne, J. Janicka, Flow Turbul. Combust. 91 (4) (2013) 867-893. http://dx.doi.org/ 10.1007/s10494-013-9492-6.

[34] P. Trisjono, K. Kleinheinz, H. Pitsch, S. Kang, Flow Turbul. Combust. 92 (1-2) (2014) 201-235. http://dx.doi.org/10.1007/s10494-013-9522-4.

[35] R.S. Barlow, M.J. Dunn, M.S. Sweeney, S. Hochgreb, Combust. Flame 159 (8) (2012) 2563-2575. http://dx.doi.org/10.1016/j.combustflame.2011.11. 013. 
[36] B. Fiorina, O. Gicquel, L. Vervisch, S. Carpentier, N. Darabiha, Combust. Flame 140 (3) (2005) 147160. http://dx.doi.org/10.1016/j.combustflame. 2004.11.002.

[37] V. Katta, W.M. Roquemore, Proc. Combust. Inst 34 (1) (2013) 1101-1108. http://dx.doi.org/10.1016/ j.proci.2012.06.033.
[38] S. Nambully, P. Domingo, V. Moureau, L. Vervisch, Combust. Flame 161 (7) (2014) 17561774. http://dx.doi.org/10.1016/j.combustflame. 2014.01.005. 